\title{
老年腹腔镜胆囊切除术中七氟烧或异丙酚复合瑞芬太尼的 临床麻醉效果观察
}

\section{Clinical Observation of Sevoflurane or Propofol Combined with Remifentanil in Elderly Patients Undergoing Aparoscopic Cholecystectomy}

\author{
王海临 \\ Hailin Wang \\ 江苏省连云港市东海县中医院 中国·江苏 连云港 222300 \\ Donghai Hospital of Traditional Chinese Medicine, Lianyungang, Jiangsu Province, 222300, China
}

\begin{abstract}
摘 要: 目的: 研究瑞芬太尼复合两种麻醉药剂的效果, 为老年腹腔镜胆囊切除术 (laparoscopic Cholecystectomy, LC) 寻求更 加安全高效的麻醉方案。方法: 以 70 例老年 LC 患者为研究对象, 分别用异丙酚、七氟烷复合瑞芬太尼麻醉, 对比麻醉效果。结 果: 两组的生命体征、苏醒及拔管情况无差异 $(P>0.05)$; 实验组使用血管活性药物的概率较对照组低 $(P<0.05)$ 。结论: 相对来 说, 七氟烷复合瑞芬太尼具有更加显著的血流动力学稳定效果。
\end{abstract}

\begin{abstract}
Objective: To study the efficacy of remifentanil in combination with two anesthetic agents, and to seek a safer and more efficient program for laparoscopic cholecystectomy (LC) in elderly patients. Methods: 70 cases of elderly LC patients were studied. Propofol and sevoflurane combined with remifentanil were used for anesthesia, and the anesthetic effect was compared. Results: there was no difference in vital signs, resuscitation and extubation between the two groups $(P>0.05)$. The probability of using vasoactive drugs in the experimental group was lower than that in the control group $(P<0.05)$. Conclusion: Sevoflurane combined with remifentanil has a more significant hemodynamic stability effect.
\end{abstract}

关键词: LC; 七氟烷; 异丙酚; 瑞芬太尼; 麻醉效果

Keywords : LC; Sevoflurane; Propofol; Remifentanil; anesthesia effect

DOI : $10.36012 /$ pmr.v2i3.2298

\section{1 引言}

在手术治疗中选取高效、安全的麻醉方案, 是十分必要 的 ${ }^{[1]}$ 。老年腹腔镜胆囊切除手术的时间较短, 受到老年患者而 受程度、体质和术后恢复等因素的影响,对于手术麻醉具有 更高的要求。当下的 LC 治疗中，基于老年患者的身体状况 等, 通常是使用瑞芬太尼等麻醉药剂, 通常是选用瑞芬太尼 复合其他药物的麻醉方案 ${ }^{[2]}$ 。针对性地分析瑞芬太尼复合不 同麻醉药物的效果, 以及安全性等相关指标, 是十分必要的, 既能够为手术治疗提供高效的麻醉方案, 又能够为麻醉方式 优化提供一定指导。研究选取 70 例老年 LC 患者,随机分组 后对比不同模式的麻醉疗效,报告如下。

【作者简介】王海临(1970 ), 男, 江苏东海人, 从事临床麻醉研究。

\section{2 资料与方法}

\section{1 研究资料}

研究于 2019 年 1 月至 2019 年 12 月选取 70 例到院接 受 LC 的老年患者开展针对性分析, 使用计算机随机分组。实 验组 35 例患者中有 21 例男性, 14 例女性, 年龄为 61 81 岁,平均 $(67.85 \pm 4.75)$ 岁, 体重 $(51.67 \pm 8.45) \mathrm{kg}$ 。对照组男 19 例, 女 16 例,年龄为 $62 \sim 80$ 岁,平均 $(67.32 \pm 5.02)$ 岁, 患者的 平均体重是 $(50.86 \pm 8.26) \mathrm{kg}$ 。两组基本信息无差异 $(P>0.05)$ 。

\section{2 研究方法}

全面监测入组患者的生命体征, 诱导麻醉后静脉持续洜 注瑞芬太尼。 
对照组静脉百注异丙酚 ( $5 \sim 10 \mathrm{mg} / \mathrm{kg} / \mathrm{h})$;

研究组持续吸入七氟烷 $(1.0 \%$ 2.0\%)。

术中依据患者实际需求应用血管活性药物, 术后关闭气 腹后停用麻醉药剂。

\section{3 观察指标}

监测生命体征, 记录术后苏醒和拔管情况, 以及血管活 性药物使用情况。

\section{4 数据处理方法}

纳人 SPSS19.0 统计软件处理数据, 用 $\left(\chi^{2}\right) 、(t)$ 检验。 $P<$ 0.05 代表结果有意义。

\section{3 结果}

\section{1 不同时间的血压和心率水平对比}

两组血压、心率水平无明显差异 $(P>0.05)$,如表 1 所示。

表 1 两组的治疗总有效对比 $[n(\%)]$

\begin{tabular}{|c|c|c|c|c|c|}
\hline 组别 & 指标 & T0 & $\mathrm{T} 1$ & $\mathrm{~T} 2$ & $\mathrm{~T} 3$ \\
\hline 守哈昍 & \multirow{4}{*}{$\begin{array}{l}\text { 收缩压 } \\
(\mathrm{mmHg})\end{array}$} & $132.56 \pm$ & $135.75 \pm$ & $127.24 \pm$ & $134.26 \pm$ \\
\hline 人地 & & 14.85 & 12.06 & 11.85 & 12.85 \\
\hline \multirow{2}{*}{ 对照组 } & & $131.45 \pm$ & $134.85 \pm$ & $126.75 \pm$ & $134.67 \pm$ \\
\hline & & 12.95 & 12.74 & 10.91 & 12.46 \\
\hline \multirow{2}{*}{ 实验组 } & \multirow{4}{*}{$\begin{array}{l}\text { 舒张压 } \\
(\mathrm{mmHg})\end{array}$} & $85.44 \pm$ & $87.75 \pm$ & $81.67 \pm$ & $88.62 \pm$ \\
\hline & & 6.75 & 7.18 & 6.74 & 7.22 \\
\hline \multirow{2}{*}{ 对照组 } & & $84.75 \pm$ & $86.95 \pm$ & $80.95 \pm$ & $87.95 \pm$ \\
\hline & & 6.45 & 6397 & 6.55 & 7.09 \\
\hline \multirow{2}{*}{ 实验组 } & \multirow{4}{*}{$\begin{array}{c}\text { 心率 } \\
\text { (次/min) }\end{array}$} & $69.75 \pm$ & $72.75 \pm$ & $67.75 \pm$ & $75.95 \pm$ \\
\hline & & 5.12 & 6.19 & 6.15 & 7.15 \\
\hline \multirow{2}{*}{ 对照组 } & & $67.54 \pm$ & $73.06 \pm$ & $68.12 \pm$ & $75.46 \pm$ \\
\hline & & 5.39 & 6.14 & 5.94 & 6.94 \\
\hline
\end{tabular}

注: * 表示与对照组相比, $P<0.05$

\section{2 手术后 OAA/S 评分、苏醒及拔管时间对比}

两组的 $\mathrm{OAA} / \mathrm{S}$ 评分无明显差异, 实验组的苏醒时间与 拔管时间略短于对照组（P>0.05), 如表 2 所示。

\section{表 2 手术后 $0 \mathrm{AA} / \mathrm{s}$ 评分、苏醒及拔管时间对比 $(\bar{x} \pm \mathrm{s})$}

\begin{tabular}{c|c|c|c|c}
\hline 组别 & 例数 & $\mathrm{OAA} / \mathrm{S}$ 评分 $($ 分) & 苏醒时间 $(\min )$ & 拔管时间 $(\mathrm{min})$ \\
\hline 实验组 & 35 & $4.28 \pm 0.51$ & $8.61 \pm 2.11$ & $10.42 \pm 2.28$ \\
\hline 对照组 & 35 & $3.96 \pm 0.49$ & $9.12 \pm 2.08$ & $11.39 \pm 2.98$ \\
\hline$t$ & & 4.226 & 5.124 & 5.338 \\
\hline$P$ & & 0.074 & 0.061 & 0.059 \\
\hline
\end{tabular}

\section{3 血管活性药物使用情况对比}

实验组血管活性药物使用率 $17.14 \%$, 对照组 $30.00 \%$, 实
验组较对照组低 $(P<0.05)$ 。

\section{4 讨论}

临床中使用麻醉药剂进行手术麻醉时, 要注意合理选择 与使用,避免使用和管理不当造成医疗差错。近些年来,医学 技术和设备不断发展进步, 腹腔镜技术不断完善与优化, LC 实施效果与价值也愈加突出, 在老年胆囊结石治疗中的应 用也明显广泛。老年患者在手术后容易发生延迟苏醒以及血 液循环稳定性差等问题。基于此,对老年患者行腹腔镜下胆 囊切除术时, 要更加谨慎地选取麻醉药剂和方式, 保证麻醉 效果。

此次研究数据显示: 两组患者生命体征均较为稳定, 且 术后苏醒及拔管情况无明显差异 $(P>0.05)$; 实验组使用血管 活性药物的概率较对照组低 $(P<0.05)$ 。相对来说, 七氟烷复 合瑞芬太尼具有更加显著的血流动力学稳定效果。瑞芬太尼 是阿片类药物, 起效迅速, 且药物半衰期短, 患者的术后苏醒 速度较快。七氟烷作为麻醉药剂不会产生气道刺激, 并且由 于不会影响心肌儿茶酚胺敏感程度, 能够使患者术中的心率 水平不受影响, 术中体征能够维持在较为稳定的状态下, 为 手术的顺利实施提供保障。老年 LC 治疗中, 选用瑞芬太尼和 七氟烷复合麻醉方案, 可以使两种药剂形成协同效应, 不仅 能够使两种麻醉药剂效果强化, 还可以促使瑞芬太尼用量降 低,有助于稳定心率和血压等生命体征。异丙酚在临床手术 治疗中的应用, 是较为广泛的, 其麻醉效果较为突出。但对于 老年 LC 患者来说, 由于身体机能和耐受性等因素的影响, 对 于手术及麻醉均有更高要求，若是选用异丙酚进行复合麻 醉, 患者的血压和心率水平受到影响而下降, 往往需要应用 对应的血管活性药物,才能够保证患者术中生命体征的稳定 性。出于老年患者手术治疗安全性等因素的考量, 七氟烷复 合麻醉能够更好地防控术中指标变化。

\section{参考文献}

[1] 邱海艳,孙亚玲,李锋.七氟烷或异丙酚复合瑞芬太尼麻醉对腹 腔镜手术患者的影响研究[J].中国处方药,2020,18(1):63-64.

[2] 张江锋, 张凯, 王更富. 老年腹腔镜胆囊切除术中七氟烷或异丙 酚复合瑞芬太尼的临床麻醉效果观察 [J]. 医学理论与实践, 2019,32(7):1027-1029. 